\title{
UV-C irradiation enhanced total phenolic contents and antioxidant capacity of Musa
}

\section{AAA 'Berangan'}

\begin{abstract}
Ultra-violet (UV) is a kind of electromagnetic radiation with wavelengths shorter than visible light. Among UV, UV-C with the wavelengths of 200-280 nm, has been used to surface sterilize food packing area, hospital and laboratories. While its usage in sterilizing drinking water has started since 1900s. Recent finding stated that UV-C is able to enhance antioxidant capacity in fruits and vegetables. Thus, a study to determine the effect of UV-C radiation on phenolic contents and antioxidant capacity of Musa AAA 'Berangan' was carried out. The mature green fruits were exposed to UV-C doses of $0,0.01,0.02,0.03$ and $0.04 \mathrm{~kJ} \mathrm{~m}-2$ and allowed to ripen at $26^{\circ} \mathrm{C}$ for 5 days after ripening initiation using $1 \mathrm{ml} \mathrm{L}-1$ ethylene. Fruit pulp of day $0,1,3$ and 5 were freeze-dried and assayed for phenolic contents and antioxidant capacity. The antioxidant capacity of the fruit was evaluated with ferric-reducing antioxidant power (FRAP), 1,1-diphenyl-2-picrylhydrazyl (DPPH) free radical-scavenging and 2,2'azinobis-(3-ethyl-benzothiazoline-6-sulfonic acid) (ABTS) assays. There was significant interaction between UV-C radiation $\times$ day after ripening on phenolic contents of 'Berangan' banana fruit. However, no significant interaction was found in antioxidant capacity of 'Berangan' banana fruit as evaluated using FRAP, DPPH and ABTS assays. Fruit irradiated with UV-C showed significant higher of phenolic contents at later stage of ripening as compared to control. There were significant differences in FRAP, ABTS and DPPH values of 'Berangan' banana fruit when exposed to different doses of UV-C. Antioxidant capacities measured with the three assays in the present study showed significant decrease as ripening day progressed. The study revealed that $0.01 \mathrm{~kJ}$ m-2 UV-C radiation is sufficient to enhance phenolic contents and antioxidant capacity.
\end{abstract}

Keyword: ABTS; DPPH; FRAP; Ripening 